\title{
An Enhancement Algorithm for Non-uniform illumination Image based on two Homomorphic Filters
}

\author{
Ruipeng Luan \\ The Chinese People's Liberation Army Unit 91550, Dalian, 116023, China \\ Irp_ph@163.com
}

Keywords: Non-uniform illumination; Image Enhancement; Homomorphic Filtering

\begin{abstract}
To solve the non-uniform illumination problem of digital images, an enhancement algorithm based on segmenting non-uniform illumination image into two sub images and filtering separately is proposed. The method how to segment the non-uniform illumination image into two sub images and fix on the cut-off frequency of homomorphic filter is studied. The experiment shows image enhancement effect of the proposed algorithm in this paper.
\end{abstract}

\section{Introduction}

In the image acquisition process, due to illumination or surface reflection causes the overall image illumination uneven, resulting in difficulties in reading information. Non-uniform illumination image is specifically manifested in the following areas: the overall gray value of the image is low, such as night image and infrared image [1][2]; image uneven illumination the image local gray value is low, unable to identify local information [3][4]; the image appears in the high light phenomenon or some image is located in the high light area [5][6]. Illumination is not all changed the original image of the image to a certain extent, therefore, the need for pre-processing. Common processing methods include gray scale transformation [7], homomorphic filter based on the illumination reflection model [8], Retinex enhancement method and gradient domain enhancement method [9][10], etc.

This paper presents a new enhancement algorithm based on two homomorphic filters, the original image is segmented into two sub images, then homomorphic filtering separately. The sub images segmentation method and homomorphic filter cut-off frequency selection method is studied. The experimental results demonstrate the effectiveness of the algorithm.

\section{Design of the Algorithm}

Step1:

Segment original image into two sub images $f_{1}, f_{2}$ using type (1). $m_{\mathrm{k}}$ is gray threshold, $n$ is the number of pixels, $d$ is control coefficient, $d=0.72$ as usual [11].

$m_{k}=d \times\left(\arg \min \left|\sum_{j=0}^{k} \frac{n_{k}}{n}-0.5\right|+0.5\right)$

The relationship between the sub images $f_{1}, f_{2}$ and the gray threshold $m_{\mathrm{k}}$ is shown in type(2):

$\left\{\begin{array}{l}f_{1}=\left\{f(i, j) \mid f \leq m_{k}\right\} \\ f_{2}=\left\{f(i, j) \mid f>m_{k}\right\}\end{array}\right.$

Step2:

The two sub images $f_{1}, f_{2}$ are equality processed separately in order to eliminate the phenomenon of over enhancement, getting new sub images $f_{1}$,,$f_{2}$, as type (3):

$\left\{\begin{array}{l}f_{1}^{\prime}=f_{1}+k \times\left(m_{k}-f_{1}\right) / n_{1} \\ f_{2}^{\prime}=f_{2}+k \times\left(f_{2}-m_{k}\right) / n_{2}\end{array}\right.$ 
Step3:

Homomorphic filter is the method of simultaneously enhancing image brightness range and image contrast in frequency domain, is represented by the incident component $i_{0}(x, y)$ and reflected components $r_{0}(x, y)$, as type (4):

$$
f(x, y)=i_{0}(x, y) \times r_{0}(x, y)
$$

Take the logarithm and Fourier transform, get type (5):

$$
z(u, v)=F_{i}(u, v) \times F_{r}(u, v)
$$

Step4:

Selection filter $H$ as type(6), $M$ and $N$ are numbers of horizontal and vertical pixels, $c$ as a constant, used to control the slope of the filter function sharpening, $c=3, D_{0}$ is cut-off frequency.

$$
\left\{\begin{array}{c}
H(u, v)=(r H-r L) \times\left(1-e^{H_{k y}}\right)+r L \\
H_{k y}=-c \times D^{2}(u, v) / D_{0}^{2} \\
D(u, v)=\sqrt{(u-M / 2)^{2}+(v-N / 2)^{2}}
\end{array}\right.
$$

Step5:

Using filter $H$ shown in type (6) to deal with the $z(u, v)$ in type (5), as type(7):

$$
S(u, v)=H(u, v) \times z(u, v)=(r H-r L) \times F_{i}^{\prime}+(r H-r L) \times F_{r}^{\prime}+r L \times Z
$$

Do Fourier inverse transform for type (7), get type (8):

$$
S(x, y)=F^{-1}(S(u, v))=(r H-r L) \times i_{o k}^{\prime}+(r H-r L) \times r_{o k}^{\prime}+r L \times\left(\ln i_{0} \times \ln r_{0}\right)
$$

Getting type (9) by exponential transformation:

$$
g=e^{s}=e^{(r H-r L) \times i_{o k}^{\prime}} \times e^{(r H-r L) \times r_{0 k}^{\prime}} \times\left(i_{0} r_{0}\right)^{r L}=i_{o k}^{r H-r L} r_{o k}^{r H-r L}\left(i_{0} r_{0}\right)^{r L}=\left(i_{o k} r_{o k}\right)^{r H} \times\left(i_{0} r_{0}\right)^{r L} /\left(i_{o k} r_{o k}\right)^{r L}
$$

Step6:

To effectively compress the dynamic range of the image, the cut-off frequency of the filter is required to be as high as possible, details of the reflection component loss should be as little as possible. The variance of the incident component and the reflection component is considered with the cut-off frequency, the variance of the reflection component is required to be as large as possible, the variance of the incident component must be within the acceptable range. According to this, we give the cut-off frequency selection method of homomorphic filter, as type (10):

$$
D_{0}=a \times(\text { median }(D)) /\left(1+e^{b(\operatorname{median}(D))}\right)
$$

In the type, $a$ is amplitude modulation factor, adjust the weight correction range; $b$ is error sensitivity factor, control sensitivity performance of cut-off frequency selection to the variance of the incident component. The function of type (10) is shown in Figure1, $a$ and $b$ take different values, $D_{0}$ corresponds to a different sensitivity region of the variance.

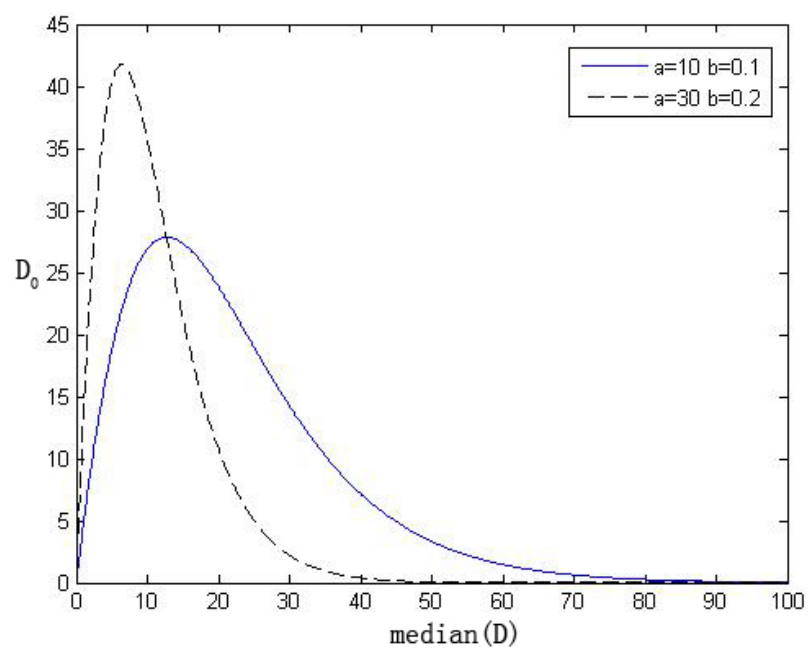

Fig.1. The function of the cut-off frequency selection method 


\section{Experiment and Results}

Experiment is carried out in the swimming pool, where indoor lighting is not open. Deploy strong light lighting equipment underwater, get non-uniform illumination original image is shown in Figure2. Segment the original image into two sub images, then homomorphic filtering separately, the sub images segmentation method and homomorphic filter cut-off frequency selection method are proposed in this paper. The experimental result is shown in Figure3, suppression of ill-posed image caused by the strong light, the dark part of the image is enhanced, can be identified as windows in swimming pool.

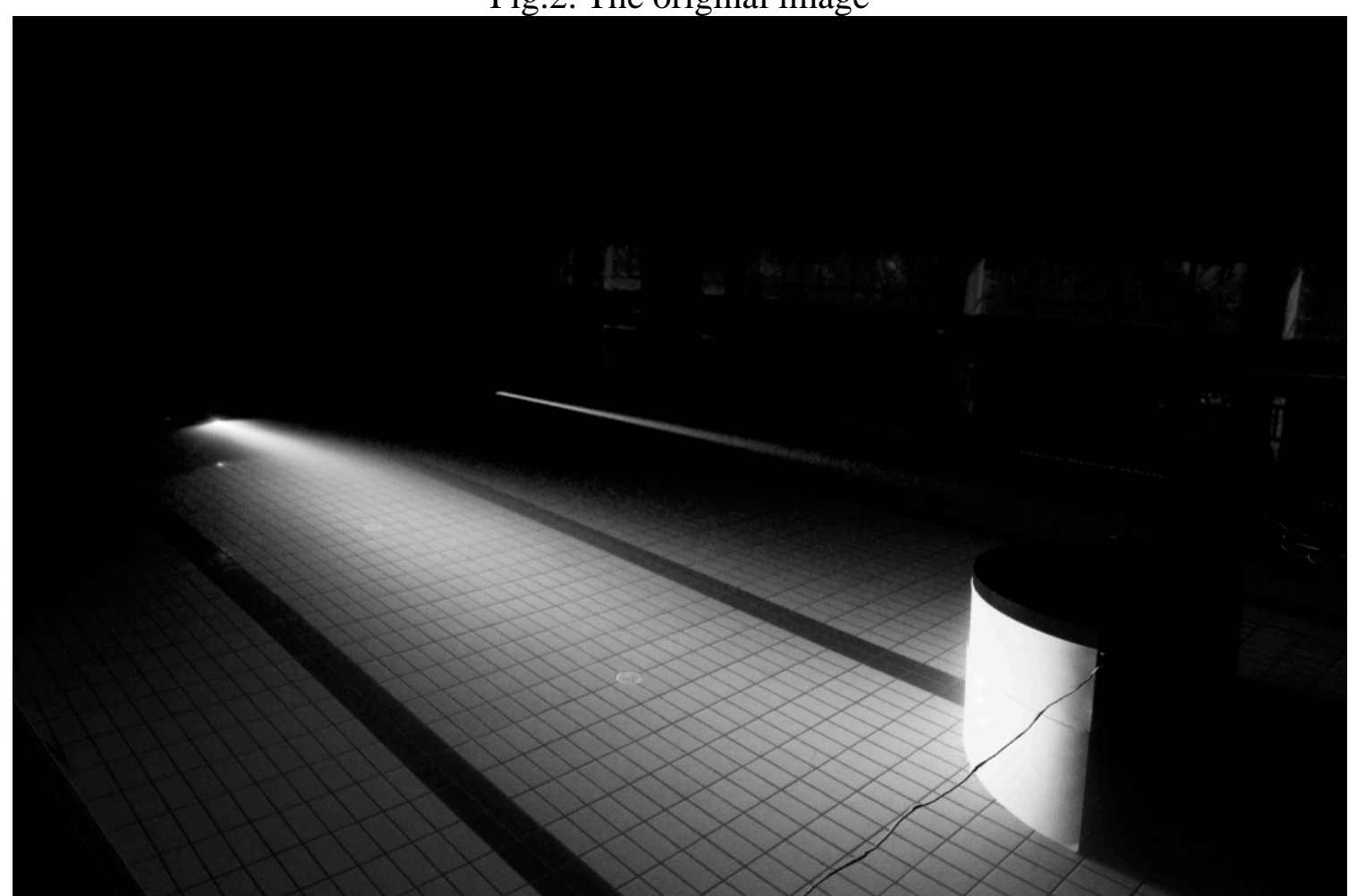

Fig.3. The experimental results 


\section{Conclusion}

To solve the non-uniform illumination problem of digital images, a new enhancement algorithm based on two homomorphic filters is proposed in this paper. The non-uniform illumination original image is segmented into two sub images, then homomorphic filtering separately. The sub images segmentation method and homomorphic filter cut-off frequency selection method is studied. The method corresponds to a different sensitivity region of the variance is especially introduced to involve homomorphic filter cut-off frequency selection problem. The experimental results demonstrate the effectiveness of the algorithm, suppression of ill-posed image caused by the strong light, the dark part of the image is enhanced. The algorithm studied in this paper is a very strong application to the image appears in the high light phenomenon or some image is located in the high light area.

\section{References}

[1] Pizer S.M, Amburn E.P. Adaptive histogram equalization and its variations[J]. Computer Vision Graphics and Image Processing, 1987, 39(3), 355-368.

[2] Kim Y.T. Contrast enhancement using brightness preserving histogram equalization [J]. IEEE Transactions on Consumer Electronics, 1997, 43(1): 1-8.

[3] Wand Y.U. Image enhancement based on equal dualistic sub-image histogram equalization method [J]. IEEE Transactions on Consumer Electronics, 1999, 45(1): 68-75.

[4] Fattal R, Llschinskid, Wermanm. Gradient domain high dynamic range compression[C]. Proc of the 29th Annual Conference on Computer Graphics and Interactive Techniques. New York: ACM Press, 2002: 249-256.

[5] Mahesh B. Chappalli, N. K. Bose. Enhanced Biggs-Andrews asymmetric iterative blind deconvolution [J]. Multidim Syst Sign Process ,2006,17:151-175.

[6] Jiang Ming, Wang Ge. Development of blind image deconvolution and its applications[J]. Journal of X-Ray Science and Technology, 2003 , 11:13-19.

[7] Chen S.D, Ramli A.R. Minimum mean brightness error histogram equalization in contrast enhancement [J]. IEEE Transactions on Consumer Electronics, 2003, 49(4): 1310-1319.

[8] Chen S.D, Ramli A.R. Contrast enhancement using recursive mean-separate histogram equalization for scalable brightness preservation [J]. IEEE Transactions on Consumer Electronics, 2003, 49(4): 1301-1309.

[9] Kim J.Y, Kim L.S. An advanced contrast enhancement using partially overlapped sub-block histogram equalization[J]. IEEE Transactions on Circuits and Systems, 2008, 11(4): 475-484.

[10] Wong A, Clausid A, Fieguth P. Adaptive Monte Carlo Retinex method for illumination and reflectance separation and color image enhancement[C]. Proc of Canadian Conference on Computer and Robot Vision. Washington DC: IEEE Computer Society, 2009: 108-115.

[11] Caselles V, Lisani J. L. Shape preserving local histogram modification[J]. IEEE Trans Image Process, 1999, 8(2):220-230. 